\title{
Spontaneous and induced activity of Heterorhabditis megidis infective juveniles during storage
}

\author{
Paul F.L. FITTERS * and Christine T. GRIFFIN \\ Institute of Bioengineering and Agroecology and Department of Biology, \\ National University of Ireland, Maynooth, Co. Kildare, Ireland
}

Received: 24 September 2004; revised: 5 November 2004 Accepted for publication: 5 November 2004

\begin{abstract}
Summary - Infective juveniles (IJ) of three Heterorhabditis megidis isolates, HF85, EU17 and UK211, were stored in water at $20^{\circ} \mathrm{C}$ for up to 10 weeks. At 2-week intervals, activity, infectivity, energy reserves and survival were measured. There was no difference between the three isolates in infectivity, which increased significantly over the first 2 weeks and declined gradually thereafter. IJ became inactive during storage. Out of storage, the highest activity was recorded in week 0 : nearly all IJ were active within the first minute of observation and remained active for the 20-min observation period. With increasing storage time, an increasing proportion of IJ were inactive in the first minute, reaching $83-96 \%$ by week 6 . The time taken by $50 \%$ of the $\mathrm{IJ}$ to become active $\left(\mathrm{AT}_{50}\right)$ initially increased with nematode age, reaching a maximum of 3-7 min in week 4 or 6 (depending on isolate) but subsequently declined to 2-4 min in week 10 . By the time the IJ were becoming more readily activated in weeks 6-8, 75\% of the lipid reserves had been depleted, and IJ had started to die. This greater propensity to become active with age may represent a switch to risk-taking behaviour in the face of impending starvation.
\end{abstract}

Keywords - activity, entomopathogenic nematode, phased infectivity, starvation, survival.

The infective juvenile (IJ) of entomopathogenic nematodes (Steinernema and Heterorhabditis) is a non-feeding stage adapted for host finding and for survival outside the host. They have considerable energy reserves, mainly neutral lipid which can constitute up to $40 \%$ of the IJ weight (Selvan et al., 1993; Abu Hatab \& Gaugler, 1997; Fitters et al., 1999). These extensive reserves enable them to survive for weeks or even months in soil or storage (Patel et al., 1997; Qiu \& Bedding, 2000; Hass et al., 2002).

It has been noted that IJ of entomopathogenic nematodes become inactive in water, presumably to conserve energy (Ishibashi \& Kondo, 1990). The propensity to become inactive in water varies between species: the percentage of IJ inactive in water was much greater for Steinernema carpocapsae than for either S. glaseri or Heterorhabditis bacteriophora (Lewis et al., 1995). Dempsey and Griffin (2002) stored H. megidis (UK211) in water at $20^{\circ} \mathrm{C}$ for 4 weeks and recorded the percentage of nematodes active in samples taken at intervals. The percentage of active IJ declined over the 4-week period but at no time did it drop below 70\% (Dempsey \& Griffin, 2002). Com- parable results were found for $S$. glaseri and $H$. bacteriophora in similar experiments (Lewis et al., 1995). This raises the question whether, and to what extent, IJ of these species become inactive when stored at room temperature for periods ranging between 1-10 weeks. The objective of both the Lewis et al. (1995) and the Dempsey and Griffin (2002) studies was to document age-related changes in the activity of IJ observed under constant conditions, and not to ascertain what proportion of IJ became inactive during storage in water. The latter is important because conserving energy by becoming inactive could extend their life span. To date the activity status of IJ undisturbed during prolonged storage has not been investigated for any species of entomopathogenic nematode.

It is well established that infective juveniles of many parasitic nematodes become inactive in the absence of stimulation and respond to a change in intensity of stimuli of various modalities (thermal, mechanical, chemical) by becoming active (Croll, 1972; Croll \& Al-Hadithi, 1972). A typical regime of activity follows: the percentage of nematodes active and the rate of undulations quickly reach a maximum, and then gradually decline. The shape

\footnotetext{
* Corresponding author, e-mail: pfitters@botanic.teagasc.ie

Present address: Teagasc, College of Amenity Horticulture, Botanic Gardens, Glasnevin, Dublin 9, Ireland.
} 
of this activity curve is different for different nematode species and depends on the type and intensity of the stimulus, the age of the nematode and the history of storage (Croll \& Al-Hadithi, 1972). A measured response is ecologically important for survival, both long term (energy conservation) and short term (quick reaction to hosts or predators).

In this study we describe the spontaneous and induced activity of stored $H$. megidis IJ. We also assess other important parameters of entomopathogenic nematodes that typically change during storage: survival, lipid reserves and infectivity (Lewis et al., 1995; Patel et al., 1997; Dempsey \& Griffin, 2002).

\section{Materials and methods}

\section{NeMATODE CULTURES}

Three north west European $H$. megidis isolates were chosen based on varied persistence and lipid depletion rates in previous experiments (Fitters \& Griffin, unpubl.). They were: UK211 (UK), HF85 (The Netherlands) and EU17 (Estonia). They were propagated in larvae of the greater wax moth, Galleria mellonella (The Mealworm Company, Sheffield, UK). Harvested IJ were washed three times by sedimentation in tap water. Nematodes $(1000 \mathrm{IJ} / \mathrm{ml})$ were stored in $5 \mathrm{~cm}$ diam. Petri dishes $\left(10 \mathrm{ml} /\right.$ dish) sealed with Parafilm ${ }^{\circledR}$. Eighteen dishes of each isolate were stored at $20^{\circ} \mathrm{C}$ in the dark in a randomised block design, each block containing one dish (replicate) per isolate. Activity, survival and infectivity were assessed after 1 day (week 0) and after 2, 4, 6, 8 and 10 weeks. Three blocks were assessed on each date. Energy reserves of IJ were assessed at weeks 0,4 and 8 . The experiment was run three times using different nematode culture batches for each experimental run.

\section{ACTIVITY}

The main objective of the study was to ascertain whether IJ that had been stored undisturbed for up to 10 weeks remained spontaneously active. A secondary question was whether the time taken by inactive IJ to become active changed over time. Disturbance was minimised by maintaining the same temperature for storage and observation. Mechanical stimulation of the nematodes and the time between storage and observation were also minimised. One dish at a time was carefully removed from the incubator, shaken once to suspend the nematodes, and
$17 \mu \mathrm{l}$ of the suspension was placed in a $16 \mathrm{~mm}^{2}$ well to facilitate simultaneous observation of all IJ in the sample (average 15; range 10-25). The well was a sub-well of a 24 well cloning plate (Sterilin, Stone, UK; 16 subwells/well). The multi-well plate was closed and placed on the stage of a dissecting microscope (Nikon SMZ-U, $4 \times$ objective) illuminated with fibre optic ('cold') light (Intralux 4000-1, Volpi, Switzerland). Room temperature was $20 \pm 1{ }^{\circ} \mathrm{C}$. The behaviour of the IJ was recorded for 20 min using a video camera mounted on the microscope. Recording began within $30 \mathrm{~s}$ of the IJ being taken from storage. Activity was assessed in min 1 (0-60 s) 5, 10, 15 and 20 . The 1 min reading gave an estimate of spontaneous activity. In each 1-min assessment period, each IJ was scored as being inactive, moderately active or highly active, based on the occurrence of the various behaviours described by Dempsey and Griffin (2002). Behaviours classed as highly active were: waving (backwards directed waves that result in forward movement), head thrusting and head lifting. Behaviours classed as moderately active were body movements (twitching and non-smooth movements), coiling and reverse wave. The latter behaviours are typical of IJ that are becoming active, although they may also be seen in fully active IJ interspersed between bouts of waving. IJ that remained inactive throughout the $20 \mathrm{~min}$ assessment period were probed with a dissecting needle. Those that failed to respond were classed as dead and were not included in the behavioural analysis.

\section{SURVIVAL, ENERGY RESERVES AND INFECTIVITY}

Following assessment of activity, the IJ suspension of the three replicate dishes was pooled. Survival of IJ was assessed from counts of three to five replicate $100 \mu \mathrm{l}$ aliquots.

Energy reserves of IJ were estimated by image analysis densitometry as described by Fitters et al. (1997). Optical density per unit area (OD units $/ \mu \mathrm{m}^{2}$ ), or mean grey level, is a good correlate of lipid levels in entomopathogenic nematodes (Fitters et al., 1997; Qiu \& Bedding, 1999). Prior to assessment, IJ were heat-killed by submersion in water at $80^{\circ} \mathrm{C}$. Measurements were performed on 30 nematodes/replicate.

Infectivity was tested using a method similar to that of Dempsey and Griffin (2002). One hundred IJ were added to the top of a $55 \mathrm{~mm}$ high vial packed with moist sand with a $G$. mellonella larva at the bottom. Vials were incubated for $24 \mathrm{~h}$ at $20^{\circ} \mathrm{C}$. The number of IJ that had invaded the insects was assessed by dissection 5 days later. There were ten replicate vials per treatment. 


\section{STATISTICS}

Statistical analyses were performed using Sigma Stat for Windows 1.0 (Jandel 1992-1994). Tests used were ANOVA (one or two-way) followed by an all pairwise multiple comparison procedure (Student-Newman-Keuls (SNK), $P<0.05$ ).

Probit analysis $\left(\right.$ SPSS $^{\circledR}$ for Windows ${ }^{\mathrm{TM}}$, Release 6.0) was used to calculate the $50 \%$ activation time $\left(\mathrm{AT}_{50}\right.$ : the time out of storage after which $50 \%$ of the IJ population was active), $50 \%$ survival time $\left(\mathrm{ST}_{50}\right)$, and the time by which $50 \%$ of available energy reserves were depleted $\left(\mathrm{ET}_{50}\right)$. Energy reserves, measured as optical density, ranged from the maximum of 0.54 OD units $/ \mu \mathrm{m}^{2}$ (week 0 ) to an asymptotic minimum of 0.36 OD units $/ \mu \mathrm{m}^{2}$. The difference between those two values, averaging 0.18 units $/ \mu \mathrm{m}^{2}$, represents available energy reserves (Fitters \& Griffin, unpubl.). $\mathrm{ET}_{50}$ and $\mathrm{AT}_{50}$ analysis was performed on the $\log _{10}$-transformed data, separated for each isolate and week $(P<0.05)$.

\section{Results}

\section{ACTIVITY}

After 1 day in storage, 95-100\% of $\mathrm{H}$. megidis IJ were active during the first minute they were observed (Fig. 1, week 0 ). In min 1, a high proportion of IJ (32-60\%) were moderately active, but after $5 \mathrm{~min}$ this behaviour was displayed by less than $16 \%$ of the population and the remainder were highly active. With increasing storage time, an increasing percentage of IJ was inactive in the first minute and a decreasing percentage of IJ became highly active at any time during the 20 min assessment. By week 6, fewer than $17 \%$ of IJ were active during the first minute and no more than $40 \%$ were highly active at any time during the observation. For each of the three isolates, the time required for $50 \%$ of IJ to become active $\left(\mathrm{AT}_{50}\right)$ tended to increase with storage time over the first 4-6 weeks, but decreased with further storage (Table 1). For both HF85 and UK211 the $\mathrm{AT}_{50}$ in week 10 was significantly lower than in week 6 (based on non-overlap of fiducial limits) showing that the nematodes became active faster in week 10 than in week 6.

Throughout the experiment, the percentage of UK211 that became active tended to be lower than for either EU17 or HF85 (Fig. 1). A more marked trend was the lower percentage of UK211 that was highly active. When stored for 4 weeks or longer, usually 40-50\% of the population of EU17 and HF85 was highly active after 20 min, while for
Table 1. Fifty percent activation time (AT50) $(95 \%$ fiducial limits) of the infective juveniles (IJ) of Heterorhabditis megidis isolates HF85, UK211 and EU17. IJ were stored at $20^{\circ} \mathrm{C}$ for up to 10 weeks prior to observation.

\begin{tabular}{lccc}
\hline $\begin{array}{l}\text { Storage time } \\
\text { (weeks) }\end{array}$ & \multicolumn{3}{c}{ AT50 (min) } \\
\cline { 2 - 4 } & EU17 & UK211 & HF85 \\
\hline 0 & $0.00^{1)}$ & $0.00^{1)}$ & $0.00^{1)}$ \\
2 & 0.77 & 1.90 & 0.55 \\
& $(0.24-1.39)$ & $(0.19-3.97)$ & $(0.25-0.89)$ \\
4 & 2.04 & 4.65 & 4.90 \\
& $(1.21-2.90)$ & $(3.48-5.92)$ & $(2.34-8.01)$ \\
6 & 3.15 & 7.29 & 3.78 \\
& $(2.02-4.37)$ & $(5.59-9.09)$ & $(3.04-4.56)$ \\
8 & 2.47 & 4.79 & 2.54 \\
& $(1.71-3.28)$ & $(3.13-6.56)$ & $(1.53-3.63)$ \\
10 & 1.90 & 4.06 & 1.94 \\
& $(1.08-2.79)$ & $(2.96-5.22)$ & $(1.25-2.71)$ \\
\hline
\end{tabular}

${ }^{1)}$ Close to $100 \%$ of IJ were active within the first minute.

UK211 this was always less than $20 \%$. The $50 \%$ activation time for UK211 tended to be longer than for HF85 or EU17, and in weeks 6 and 10 the difference between UK211 and the other two isolates was significant based on the non-overlap of $95 \%$ fiducial limits (Table 1).

\section{SURVIVAL, ENERGY RESERVES AND INFECTIVITY}

More than $80 \%$ of the IJ of each isolate remained alive in week 6 (Fig. 2). At weeks 8 and 10, there were significantly $(P<0.05)$ fewer HF85 IJ alive compared to UK211 and EU17, and this is reflected in a significantly shorter $\mathrm{ST}_{50}$ for this isolate (Table 2).

The time taken for $50 \%$ of the reserves to be depleted $\left(\mathrm{ET}_{50}\right)$ ranged from 3.2 to 5.4 weeks (Table 2). HF85 had the lowest $\mathrm{ET}_{50}$ reflecting more rapid depletion of reserves but differences between isolates were not significant as the fiducial limits overlapped (Table 2). By week 8 , an estimated $75 \%$ or more of the energy reserves of all three isolates were depleted (data not shown).

The three isolates did not differ in infectivity at any assessment date (ANOVA, $P>0.05$ ). Overall, infectivity showed a significant two-fold increase during the first 2 weeks of storage and declined thereafter (Fig. 3).

\section{Discussion}

This is the first attempt to document the degree to which the IJ of a species of entomopathogenic nematode remain spontaneously active when stored undisturbed in water 


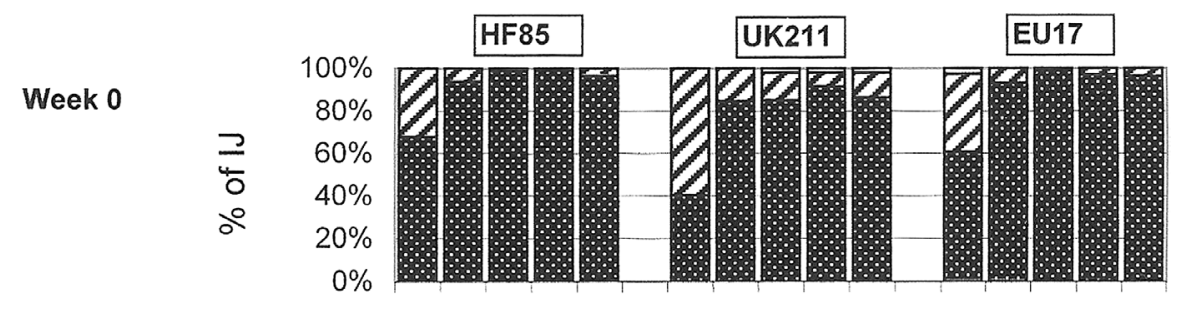

Week 2

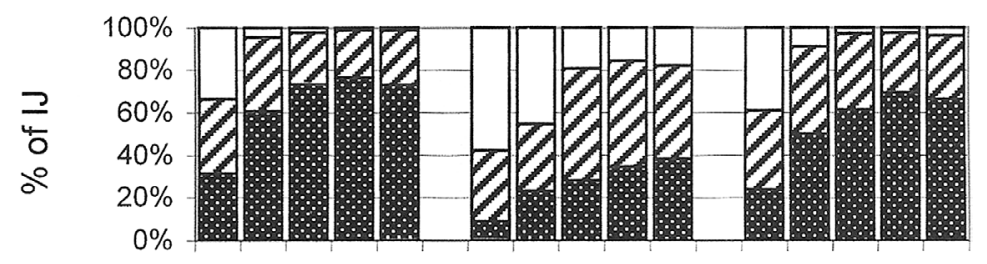

Week 4

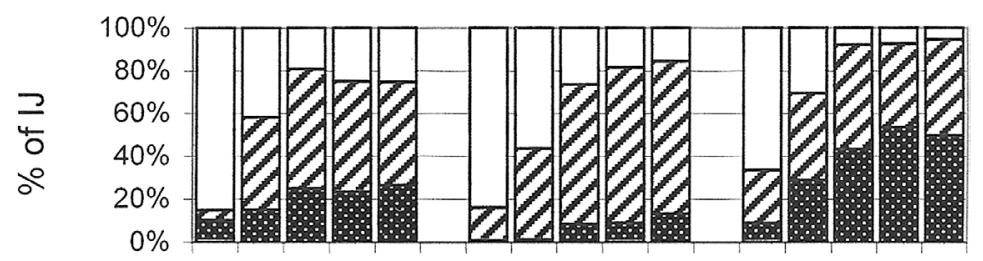

Week 6

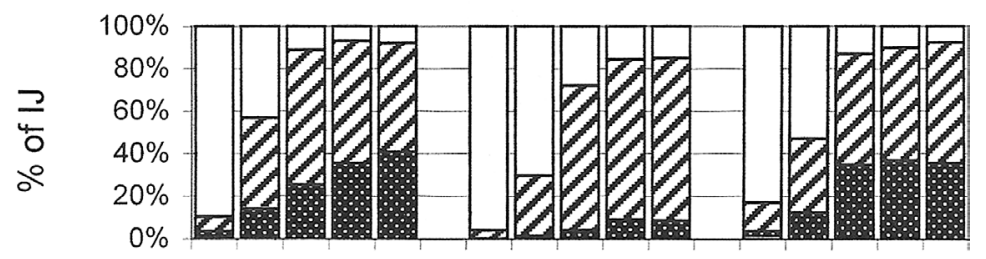

Week 8

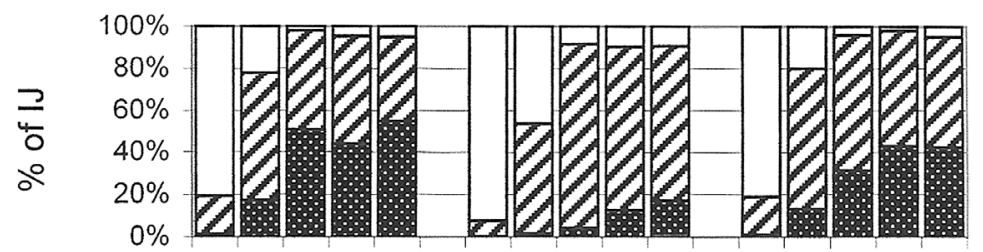

Week 10

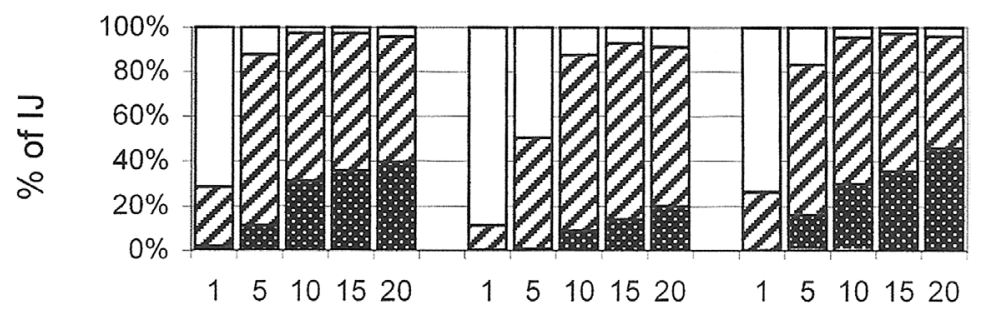

$\checkmark$ Moderately active

圈 Highly active

Time out of storage ( $\mathrm{min}$ )

\section{口inactive}

Fig. 1. Percentage of highly active, moderately active and inactive living infective juveniles of Heterorhabditis megidis isolates HF85, UK211 and EU17 during the first 20 min after removal from storage at weeks 0, 2, 4, 6, 8 and 10. Each bar represents the mean of three experiments. 


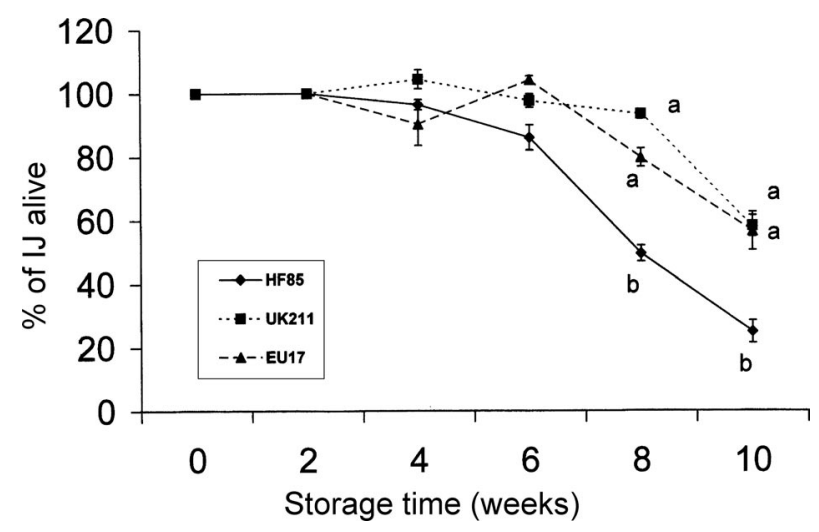

Fig. 2. Percentage of IJ alive of Heterorhabditis megidis isolates HF85, UK211 and EU17 during storage in tap water at $20^{\circ} \mathrm{C}$ for 10 weeks. Within weeks, same letters indicate no significant difference between isolates (ANOVA, SNK, $\mathrm{P}<0.05)$. Each point represents the mean of three experiments (with standard error of mean).

Table 2. Fifty percent survival time $\left(S T_{50}\right)$ and $50 \%$ energy depletion point (ET 50 ) (95\% fiducial limits) of north west European Heterorhabditis megidis isolates HF85, UK211 and EU17 stored in water at $20^{\circ} \mathrm{C}$.

\begin{tabular}{lcc}
\hline Isolate & $\mathrm{ST}_{50}$ (weeks) & $\mathrm{ET}_{50}$ (weeks) \\
\hline HF85 & $8.26(7.92-8.64)$ & $3.21(1.42-4.72)$ \\
UK211 & $10.49(10.03-11.21)$ & $5.36(4.36-6.51)$ \\
EU17 & $10.95(8.69-33.38)$ & $4.45(3.56-5.37)$ \\
\hline
\end{tabular}

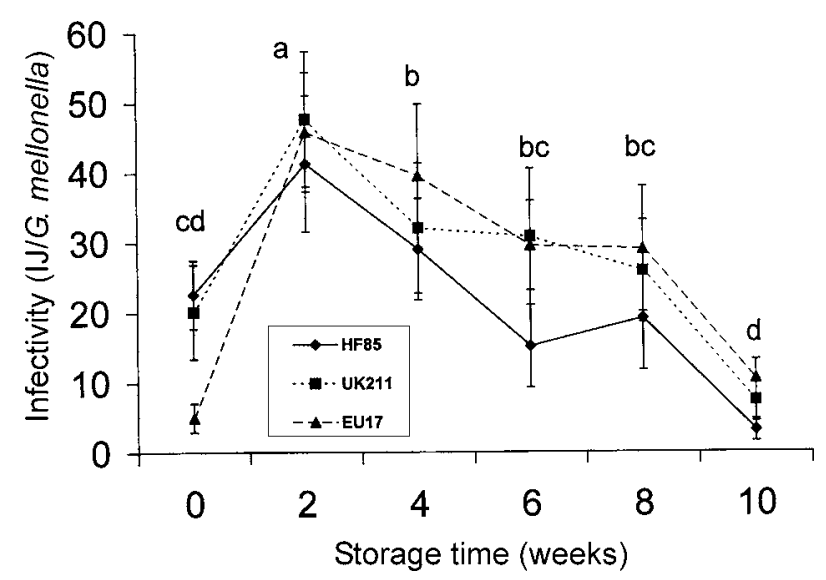

Fig. 3. Infectivity of Heterorhabditis megidis isolates HF85, UK211 and EU17 for Galleria mellonella in sand. Nematode infective juveniles were stored in tap water at $20^{\circ} \mathrm{C}$ for up to 10 weeks. Within weeks, same letters indicate no significant difference between isolates (ANOVA, SNK, $\mathrm{P}<0.05$ ). Each point represents the mean of three experiments (with standard error of mean). for several weeks. We show that $H$. megidis IJ become inactive during storage in water, even at the relatively high storage temperature of $20^{\circ} \mathrm{C}$. It is clear that from week 4 onwards, a minority $(<40 \%)$ of the IJ were active when first observed. This may represent an overestimate of the level of spontaneous activity: IJ were clearly stimulated into activity, presumably mainly due to the mechanical stimulation of shaking and pipetting or by light exposure.

After just 1 day (week 0), all IJ were active during the first minute of observation. Either they had not become inactive at all during overnight storage, or they were very readily activated. With increasing storage time, a greater percentage of IJ was inactive during the first minute of observation, and they took longer to become active. IJ of entomopathogenic nematodes generally become less active with age, whether activity is measured as percentage active, rate of movement or extent of dispersal (Lewis et al., 1995; Westerman, 1997; Cohen et al., 2002; Dempsey \& Griffin, 2002). Other time-dependent factors such as a progressively increasing interval since last stimulation, or progressive changes in the chemical or microbial composition of the surrounding medium, may, in addition of ageing, also have contributed to the changed behaviour of the IJ in addition to ageing.

If inactivity is an energy-saving strategy aimed at prolonging survival, then it might be expected that UK211, which was the least readily activated of the three isolates, would utilise its reserves more slowly and survive longer than the other two isolates. UK211 did lose its energy reserves more slowly (although not significantly) than either EU17 or HF85, and survived significantly longer than HF85 (although not longer than EU17). The contribution of activity to energy expenditure in nematodes is unresolved (see Wright, 1998). However, rate of activity of IJ (measured as waves/min) explained $60 \%$ of the variation between $H$. megidis isolates in the rate at which lipids were depleted (Fitters \& Griffin, unpubl.), implicating activity in energy depletion for these nematodes.

The increase in infectivity of each of the three $H$. megidis isolates during 2 weeks of storage at $20^{\circ} \mathrm{C}$ confirms the phenomenon for the UK211 strain of H. megidis, as previously reported by Griffin (1996), Dempsey and Griffin (2002) and Ryder and Griffin (2003). We also extend the finding for two geographically distinct isolates, HF85 from The Netherlands and EU17 from Estonia. Each of these isolates showed the same pattern of infectivity, indicating that the evolutionary pressures on this trait have been similar for all three isolates but, as all three had been in culture for several years prior to this investigation, in- 
advertent laboratory selection may have contributed to the homogenisation of this trait. The phenomenon of 'phased infectivity' in entomopathogenic nematodes is controversial (see e.g., Campbell et al., 1999; Ryder \& Griffin, 2003) but the present results confirm that the proportion of $H$. megidis invading hosts in a time limited-assay undergoes a reproducible increase before declining.

Freshly harvested (week 0) IJ were nearly all active when first observed. The highly active nature of $H$. megidis immediately after emerging from the cadaver may reflect the need to disperse rapidly from the highly crowded environs of the cadaver. Dempsey and Griffin (2002) showed that, during the first week after emergence, H. megidis UK211 had high dispersal rates coupled with low infectivity. This dispersive phase would ensure that not all IJ would infect local hosts, but also hosts further away, and thus avoid overcrowding. Dempsey and Griffin (2002) described the activity of $H$. megidis over a 4-week period. They distinguished three phases: an initial highly active phase, an infective phase, and a third phase during which infectivity and activity were expressed at a lower level. As our observations extended over a longer period it is possible to distinguish a further change in behaviour of H. megidis. After 6-8 weeks storage, the IJ were more easily activated than in the previous observation period (weeks 4 or 6 , respectively) (Table 1 ). The reduction in activation time could be a result of selective mortality of less active IJ; however, at most $20 \%$ of the IJ had died by the time the decrease in $\mathrm{AT}_{50}$ was recorded. The alternative explanation is that it represents an increased propensity of the general population to become active. Possibly, as lipids and other reserves are depleted, IJ switch to a more active behaviour, representing a switch to risktaking behaviour in the face of starvation (McNamara \& Houston, 1991). The differences between isolates in the time at which they became more easily activated is consistent with either explanation: it occurred earlier (week 6) for HF85 than for the other two isolates, and HF85 also both experienced earlier mortality and used up its reserves faster.

The ultimate goal of IJ is to infect, but despite the increased propensity to become active after 6-8 weeks, their capacity to infect continued to decline (Fig. 3). This could be a result of the decreasing energy reserves, becoming insufficient for reaching and/or entering the host insect.

\section{Acknowledgements}

The research was funded by the Programme for Research in Third Level Institutions (PRTLI) of the Higher Education Authority, Ireland. We are grateful to Dr Catherine Dempsey for her useful comments on the manuscript.

\section{References}

Abu Hatab, M. \& GaUgler, R. (1997). Influence of growth temperature on fatty acids and phospholipids of Steinernema riobravis infective juveniles. Journal of Thermal Biology 22, 237-244.

Campbell, J.F., Koppenhöfer, A.M., Kaya, H.K \& ChInNASRI, B. (1999). Are there temporarily non-infectious dauers in entomopathogenic nematode populations: a test of the phased infectivity model. Parasitology 118, 499-508.

Cohen, N.E., Brown, I.M. \& Gaugler, R. (2002). Physiological ageing and behavioural plasticity of Heterorhabditis bacteriophora infective juveniles. Nematology 4, 81-87.

Croll, N.A. (1972). Behavioural activities of nematodes. Helminthological Abstracts, Series A 41, 359-377.

Croll, N.A. \& Al-Hadithi, I. (1972). Sensory basis of activity in Ancylostoma tubaeforme infective larvae. Parasito$\operatorname{logy} 64,279-291$.

Dempsey, C.M. \& GRIFFIn, C.T. (2002). Phased activity in Heterorhabditis megidis infective juveniles. Parasitology 124, 605-613.

Fitters, P.F.L., Wright, D.J., Meijer, E.M.J. \& GrifFIN, C.T. (1997). Estimation of lipid reserves in unstained living and dead nematodes by image analysis. Journal of $\mathrm{Ne}$ matology 29, 160-167.

FitTers, P.F.L., PATEl, M.N., Griffin, C.T. \& Wright, D.J. (1999). Fatty acid composition of Heterorhabditis sp. during storage. Comparative Biochemistry and Physiology B 124, 81-88.

GRIFFIN, C.T. (1996). Effects of prior storage conditions on the infectivity of Heterorhabditis sp. (Nematoda: Heterorhabditidae). Fundamental and Applied Nematology 19, 95-102.

Hass, B., Downes, M.J. \& GRIFFIn, C.T. (2002). Persistence of four Heterorhabditis spp. isolates in soil: role of lipid reserves. Journal of Nematology 34, 151-158.

IsHIBASHI, N. \& Kondo, E. (1990). Behaviour of infective juveniles. In: Gaugler, R. \& Kaya, H. (Eds). Entomopathogenic nematodes in biological control. California, USA, CRC Press, pp. 139-150.

Lewis, E.E., Selvan, S., Campbell, J.F. \& Gaugler, R. (1995). Changes in foraging behaviour during the infective stage of entomopathogenic nematodes. Parasitology 110, 583-590. 
McNamara, J.M. \& Houston, A.I. (1991). Starvation and predation in a patchy environment. In: Shorrocks, B. \& Swingland, I.R. (Eds). Living in a patchy environment. Oxford, UK, Oxford University Press, pp. 23-43.

Patel, M.N., Stolinski, M. \& Wright, D.J. (1997). Neutral lipids and the assessment of infectivity in entomopathogenic nematodes: observations on four Steinernema species. Parasitology 114, 489-496.

QIU, L. \& BEDDING, R. (1999). A rapid method for the estimation of mean dry weight and lipid content of the infective juveniles of entomopathogenic nematodes using image analysis. Nematology 1, 655-660.

QIU, L. \& BEDDING, R. (2000). Energy metabolism and its relation to survival and infectivity of infective juveniles of Steinernema carpocapsae under aerobic conditions. Nematology 2, 551-559.
Ryder, J.J. \& GRIFFIn, C.T. (2003). Phased infectivity in Heterorhabditis megidis: the effects of infection density in the parental host and filial generation. International Journal of Parasitology 33, 1013-1018.

Selvan, S., GAugler R. \& Lewis, E.E. (1993). Biochemical energy reserves of entomopathogenic nematodes. Journal of Parasitology 79, 167-172.

Westerman, P.R. (1997). Comparative vertical migration of twenty one isolates of insect parasitic nematodes Heterorhabditis spp. in sand at $20^{\circ} \mathrm{C}$. Fundamental and Applied Nematology 18, 149-158.

WRIGHT, D.J. (1998). Respiratory physiology, nitrogen excretion and osmotic and ionic regulation. In: Perry, R.N. \& Wright, D.J. (Eds). The physiology and biochemisty of free-living and plant-parasitic nematodes. Wallingford, UK, CABI Publishing, pp. 103-131. 
Copyright of Nematology is the property of VSP International Science Publishers and its content may not be copied or emailed to multiple sites or posted to a listserv without the copyright holder's express written permission. However, users may print, download, or email articles for individual use. 